Journal OF COMBINATORICS

Volume 0, Number 0, 1-8, 0000

\title{
Line configurations and $r$-Stirling partitions
}

\author{
Brendon Rhoades and Andrew Timothy Wilson
}

\begin{abstract}
A set partition of $[n]:=\{1,2, \ldots, n\}$ is called $r$-Stirling if the numbers $1,2, \ldots, r$ belong to distinct blocks. Haglund, Rhoades, and Shimozono constructed a graded ring $R_{n, k}$ depending on two positive integers $k \leq n$ whose algebraic properties are governed by the combinatorics of ordered set partitions of $[n]$ with $k$ blocks. We introduce a variant $R_{n, k}^{(r)}$ of this quotient for ordered $r$-Stirling partitions which depends on three integers $r \leq k \leq n$. We describe the standard monomial basis of $R_{n, k}^{(r)}$ and use the combinatorial notion of the coinversion code of an ordered set partition to reprove and generalize some results of Haglund et. al. in a more direct way. Furthermore, we introduce a variety $X_{n, k}^{(r)}$ of line configurations whose cohomology is presented as the integral form of $R_{n, k}^{(r)}$, generalizing results of Pawlowski and Rhoades.
\end{abstract}

\section{Introduction}

Given two integers $r \leq n$, a set partition of $[n]:=\{1,2, \ldots, n\}$ is called $r$-Stirling if the first $r$ letters $1,2, \ldots, r$ lie in distinct blocks. The $r$-Stirling number (of the second kind) $\operatorname{Stir}_{n, k}^{(r)}$ counts $r$-Stirling partitions of $[n]$ with $k$ blocks. An ordered $r$-Stirling partition is an $r$-Stirling partition $\sigma=\left(B_{1} \mid\right.$ $\cdots \mid B_{k}$ ) equipped with a total order on its blocks. We let $\mathcal{O P}_{n, k}^{(r)}$ denote the family of ordered $r$-Stirling partitions of $[n]$ with $k$ blocks; these are counted by $\left|\mathcal{O} \mathcal{P}_{n, k}^{(r)}\right|=k ! \cdot \operatorname{Stir}_{n, k}^{(r)}$.

An example element of $\mathcal{O P}_{7,4}^{(3)}$ is $(26|5| 17 \mid 34)$. On the other hand, the ordered set partition (45|2|136|7) fails to be 3-Stirling since 1 and 3 belong to the same block. The symmetric group $S_{n}$ acts on ordered set partitions of $[n]$ by letter permutation. Although $\mathcal{O} \mathcal{P}_{n, k}^{(r)}$ is not closed under the full action of $S_{n}$, it does carry an action of the parabolic subgroup $S_{r} \times S_{n-r}$.

When $r=k=n$, an element of $\mathcal{O P}_{n, n}^{(n)}$ is just a permutation in $S_{n}$. The combinatorics of the symmetric group $S_{n}$ is well-known to govern both the

arXiv: http://arxiv.org/abs/1804.07879 
algebraic structure of the coinvariant ring $R_{n}$ and the geometric structure of the flag variety $\mathcal{F} \ell(n)$.

In the case $r=0$ where $\mathcal{O P} \mathcal{P}_{n, k}:=\mathcal{O P}_{n, k}^{(0)}$ is the collection of $k$-block ordered set partitions of $[n]$, the Delta Conjecture [2] in the theory of Macdonald polynomials motivated the definition and study of a generalized coinvariant ring $R_{n, k}$ [3] and a generalization $X_{n, k}$ of the flag variety [5] which specialize to their classical counterparts when $k=n$. The algebraic properties of $R_{n, k}$ and the geometric properties of $X_{n, k}$ are governed by combinatorial properties of ordered set partitions in $\mathcal{O P} \mathcal{P}_{n, k}$.

At a workshop in Montréal in the Summer of 2017, Jeff Remmel asked the authors if it was possible to extend this theory to encapsulate ordered $r$-Stirling partitions; in this paper we do exactly that. We consider a quotient ring $R_{n, k}^{(r)}$ and a variety $X_{n, k}^{(r)}$ whose properties are controlled by the combinatorics of $\mathcal{O} \mathcal{P}_{n, k}^{(r)}$. The quotient $R_{n, k}^{(r)}$ of $\mathbb{Q}\left[\mathbf{x}_{n}\right]:=\mathbb{Q}\left[x_{1}, \ldots, x_{n}\right]$ (together with its companion quotient $S_{n, k}^{(r)}$ of $\left.\mathbb{Z}\left[\mathbf{x}_{n}\right]:=\mathbb{Z}\left[x_{1}, \ldots, x_{n}\right]\right)$ is defined as follows. If $\mathbf{x}_{m}=\left(x_{1}, \ldots, x_{m}\right)$ is a list of variables and $d \geq 0$, we recall the elementary and homogeneous symmetric polynomials of degree $d$ in the variable set $\mathbf{x}_{m}$ :

$$
\begin{aligned}
e_{d}\left(\mathbf{x}_{m}\right) & :=\sum_{1 \leq i_{1}<\cdots<i_{d} \leq m} x_{i_{1}} \cdots x_{i_{d}} \\
h_{d}\left(\mathbf{x}_{m}\right) & :=\sum_{1 \leq i_{1} \leq \cdots \leq i_{d} \leq m} x_{i_{1}} \cdots x_{i_{d}} .
\end{aligned}
$$

Definition 1.1. For $r \leq k \leq n$, let $I_{n, k}^{(r)} \subseteq \mathbb{Q}\left[\mathbf{x}_{n}\right]$ be the ideal

$$
I_{n, k}^{(r)}:=\left\langle\begin{array}{c}
x_{1}^{k}, x_{2}^{k}, \ldots, x_{n}^{k}, \\
e_{n}\left(\mathbf{x}_{n}\right), e_{n-1}\left(\mathbf{x}_{n}\right), \ldots, e_{n-k+1}\left(\mathbf{x}_{n}\right), \\
h_{k-r+1}\left(\mathbf{x}_{r}\right), h_{k-r+2}\left(\mathbf{x}_{r}\right), \ldots, h_{k}\left(\mathbf{x}_{r}\right)
\end{array}\right\rangle
$$

and let $R_{n, k}^{(r)}$ be the corresponding quotient ring:

$$
R_{n, k}^{(r)}:=\mathbb{Q}\left[\mathbf{x}_{n}\right] / I_{n, k}^{(r)}
$$

Furthermore, let $J_{n, k}^{(r)} \subseteq \mathbb{Z}\left[\mathbf{x}_{n}\right]$ be the ideal in $\mathbb{Z}\left[\mathbf{x}_{n}\right]$ with the same generating set as $I_{n, k}^{(r)}$ and let $S_{n, k}^{(r)}=\mathbb{Z}\left[\mathbf{x}_{n}\right] / J_{n, k}^{(r)}$ be the corresponding quotient. 


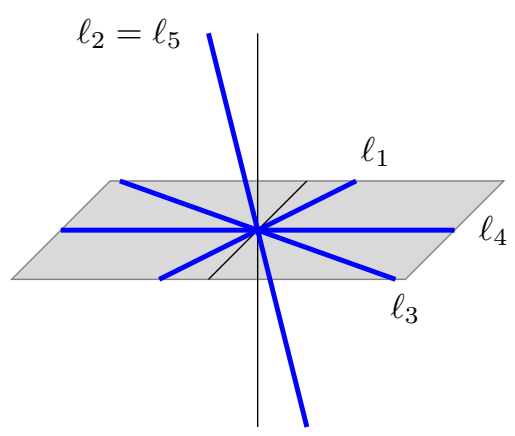

Figure 1: A point in $X_{5,3}^{(2)}$.

When $r=k=n$, the ideal $I_{n}:=I_{n, n}^{(n)}$ is just the classical invariant ideal $\left\langle e_{1}\left(\mathbf{x}_{n}\right), e_{2}\left(\mathbf{x}_{n}\right), \ldots, e_{n}\left(\mathbf{x}_{n}\right)\right\rangle$ generated by the $n$ elementary symmetric polynomials. When $r=0$, the ideal $I_{n, k}:=I_{n, k}^{(0)}$ is precisely the ideal considered in [3], and its companion ideal $J_{n, k}:=J_{n, k}^{(0)}$ over the ring of integers was considered in [5].

The quotient ring $S_{n, k}^{(r)}$ will be shown to calculate the cohomology (singular, with coefficients in $\mathbb{Z}$ ) of a natural space $X_{n, k}^{(r)}$ whose geometry is governed by the combinatorics of $\mathcal{O} \mathcal{P}_{n, k}^{(r)}$. Let $\mathbb{P}^{k-1}$ be the complex projective space of lines through the origin in $\mathbb{C}^{k}$, so that $\left(\mathbb{P}^{k-1}\right)^{n}$ is the complex algebraic variety of all $n$-tuples $\left(\ell_{1}, \ldots, \ell_{n}\right)$ of lines through the origin in $\mathbb{C}^{k}$. We consider the following family of line configurations.

Definition 1.2. Let $r \leq k \leq n$ and define a subset $X_{n, k}^{(r)} \subseteq\left(\mathbb{P}^{k-1}\right)^{n}$ by

$$
X_{n, k}^{(r)}:=\left\{\left(\ell_{1}, \ell_{2}, \ldots, \ell_{n}\right) \in\left(\mathbb{P}^{k-1}\right)^{n}: \begin{array}{c}
\ell_{1}+\ell_{2}+\cdots+\ell_{n}=\mathbb{C}^{k} \text { and } \\
\operatorname{dim}\left(\ell_{1}+\ell_{2}+\cdots+\ell_{r}\right)=r
\end{array}\right\}
$$

A typical point in $X_{n, k}^{(r)}$ is an $n$-tuple of lines $\left(\ell_{1}, \ldots, \ell_{n}\right)$ through the origin in $\mathbb{C}^{k}$ such that these lines span $\mathbb{C}^{k}$ and such that the first $r$ of these lines are linearly independent. An example of such a line configuration in $X_{5,3}^{(2)}$ is shown in Figure 1; the first two lines $\ell_{1}$ and $\ell_{2}$ are linearly independent, and the five lines $\ell_{1}, \ldots, \ell_{5}$ together span $\mathbb{C}^{3}$.

The product group $S_{r} \times S_{n-r}$ acts on $X_{n, k}^{(r)}$ by line permutation. The set $X_{n, k}^{(r)}$ is a Zariski open subset of $\left(\mathbb{P}^{k-1}\right)^{n}$ and is therefore both a variety and a smooth complex manifold. 
When $r=k=n$, the space $X_{n, k}^{(r)}$ may be identified with the quotient $G / T$, where $G=G L_{n}(\mathbb{C})$ is the group of invertible $n \times n$ complex matrices and $T \subseteq G$ is the diagonal torus. If $B \subseteq G$ is the Borel subgroup of upper triangular matrices, the quotient $G / B$ is the classical flag variety $\mathcal{F} \ell(n)$ of type $\mathrm{A}_{n-1}$ and the canonical projection $G / T \rightarrow G / B$ is a homotopy equivalence. When $r=0$, the space $X_{n, k}:=X_{n, k}^{(0)}$ of $n$-tuples of lines spanning $\mathbb{C}^{k}$ was defined and studied by Pawlowski and Rhoades as an extension of the flag variety [5].

The remainder of the paper is organized as follows. In Section 2 we will introduce a new statistic on an ordered set partition $\sigma$ : the coinversion code $\operatorname{code}(\sigma)$. This will allow us to read off the standard monomial basis of the quotient ring $R_{n, k}^{(r)}$ directly from the combinatorics of $\mathcal{O} \mathcal{P}_{n, k}^{(r)}$, both extending and making more combinatorial the results regarding $R_{n, k}$ in [3]. In Section 3 we will study the space of line configurations $X_{n, k}^{(r)}$ and prove that $H^{\bullet}\left(X_{n, k}^{(r)}\right)=S_{n, k}^{(r)}$. We will also describe an affine paving of $X_{n, k}^{(r)}$ with cells indexed by partitions in $\mathcal{O} \mathcal{P}_{n, k}^{(r)}$, together with formulas for the representatives of the closures of these cells in cohomology.

\section{Coinversion codes and standard bases}

Recall that an inversion of a permutation $w \in S_{n}$ is a pair $1 \leq i<j \leq n$ such that $i$ appears to the right of $j$ in the one-line notation $w=w_{1} \ldots w_{n}$, so that the inversions of $231 \in S_{3}$ are the pairs $(1,2)$ and $(1,3)$. Extending this notion to ordered set partitions, if $\sigma=\left(B_{1}|\cdots| B_{k}\right)$ is an ordered set partition of $[n]$ with $k$ blocks, a pair $1 \leq i<j \leq n$ is said to be an inversion of $\sigma$ if

- the block of $i$ is strictly to the right of the block of $j$ in $\sigma$, and

- the letter $i$ is minimal in its block.

We let $\operatorname{inv}(\sigma)$ be the number of inversions of $\sigma$, so that if $\sigma=(25|1| 34) \in$ $\mathcal{O P} \mathcal{P}_{5,3}$ the inversion pairs are $(1,2),(1,5)$, and $(3,5)$ so that $\operatorname{inv}(\sigma)=3$.

We will not be interested in the statistic inv itself, but rather its complementary statistic. For any three integers $r \leq k \leq n$, it is not hard to see that the statistic inv on $\mathcal{O P}_{n, k}^{(r)}$ achieves its maximum value at the unique point $\sigma_{0}:=(k, k+1 \ldots, n-1, n|k-1| \cdots \mid 1) \in \mathcal{O P}_{n, k}^{(r)}$, and that

$$
\operatorname{inv}\left(\sigma_{0}\right)=(n-k)(k-1)+\left(\begin{array}{l}
k \\
2
\end{array}\right) .
$$


We define the statistic coinv on $\mathcal{O P} \mathcal{P}_{n, k}^{(r)}$ by the rule

$$
\operatorname{coinv}(\sigma):=(n-k)(k-1)+\left(\begin{array}{l}
k \\
2
\end{array}\right)-\operatorname{inv}(\sigma)
$$

For example, we have

$\operatorname{coinv}(25|1| 34)=(5-3)(3-1)+\left(\begin{array}{l}3 \\ 2\end{array}\right)-\operatorname{inv}(25|1| 34)=4+3-3=4$.

It will be convenient to break up the coinversion statistic coinv into a sequence of smaller statistics. Given an ordered set partition $\sigma=\left(B_{1} \mid\right.$ $\left.\cdots \mid B_{k}\right) \in \mathcal{O P}_{n, k}^{(r)}$, define the coinversion code $\operatorname{code}(\sigma)=\left(c_{1}, c_{2}, \ldots, c_{n}\right)$ as follows. Suppose $1 \leq i \leq n$ and $i \in B_{j}$. Then

$$
c_{i}= \begin{cases}\left|\left\{\ell>j: \min \left(B_{\ell}\right)>i\right\}\right| & \text { if } i=\min \left(B_{j}\right) \\ \left|\left\{\ell>j: \min \left(B_{\ell}\right)>i\right\}\right|+(j-1) & \text { if } i \neq \min \left(B_{j}\right) .\end{cases}
$$

The coinversion code of $(25|1| 34)$ is therefore code $(\sigma)=\left(c_{1}, c_{2}, c_{3}, c_{4}, c_{5}\right)=$ $(1,1,0,2,0)$. The coinversion code breaks the statistic coinv into pieces.

Proposition 2.1. Let $\sigma \in \mathcal{O P}_{n, k}^{(r)}$ with $\operatorname{code}(\sigma)=\left(c_{1}, c_{2}, \ldots, c_{n}\right)$. Then

$$
\operatorname{coinv}(\sigma)=c_{1}+c_{2}+\cdots+c_{n} .
$$

Which sequences $\left(c_{1}, c_{2}, \ldots, c_{n}\right)$ of nonnegative integers can arise as the coinversion code of some element $\sigma \in \mathcal{O P}_{n, k}^{(r)}$ ? When $r=k=n$, these are precisely the sequences $\left(c_{1}, c_{2}, \ldots, c_{n}\right)$ which are componentwise $\leq$ the staircase $(n-1, n-2, \ldots, 0)$ of length $n$. To state the answer for general $r \leq k \leq n$, we will need some definitions.

If $S=\left\{s_{1}<s_{2}<\cdots<s_{m}\right\}$ is any subset of $[n]$, the skip composition $\gamma(S)=\left(\gamma(S)_{1}, \ldots, \gamma(S)_{n}\right)$ is the sequence given by

$$
\gamma(S)_{i}= \begin{cases}i-j+1 & \text { if } i=s_{j} \in S \\ 0 & \text { if } i \notin S .\end{cases}
$$

We also let $\gamma(S)^{*}=\left(\gamma(S)_{n}, \ldots, \gamma(S)_{1}\right)$ be the reversal of the skip composition. As an example, if $n=7$ and $S=\{2,3,6\}$ then $\gamma(S)=(0,2,2,0,0,4,0)$ and $\gamma(S)^{*}=(0,4,0,0,2,2,0)$.

Theorem 2.2. Let $r \leq k \leq n$. The map $\sigma \mapsto \operatorname{code}(\sigma)$ gives a bijection from $\mathcal{O} \mathcal{P}_{n, k}^{(r)}$ to the family $\left(c_{1}, \ldots, c_{n}\right)$ of nonnegative integer sequences such that 
- for all $r+1 \leq i \leq n$ we have $c_{i}<k$,

- for all $1 \leq i \leq r$ we have $c_{i}<k-i+1$, and

- for any subset $S \subset[n]$ with $|S|=n-k+1$, the componentwise inequality $\gamma(S)^{*} \leq\left(c_{1}, \ldots, c_{n}\right)$ fails to hold.

Proof. Let $\mathcal{C}_{n, k}^{(r)}$ be the family of length $n$ sequences of nonnegative integers which satisfy the three conditions in the statement of the theorem. Let $\sigma \in \mathcal{O} \mathcal{P}_{n, k}^{(r)}$ with $\operatorname{code}(\sigma)=\left(c_{1}, \ldots, c_{n}\right)$. We show that $\left(c_{1}, \ldots, c_{n}\right) \in \mathcal{C}_{n, k}^{(r)}$, so that the function code $: \mathcal{O} \mathcal{P}_{n, k}^{(r)} \rightarrow \mathcal{C}_{n, k}^{(r)}$ is well-defined. This is verified as follows.

- For any $1 \leq i \leq n$, the block $B$ of $\sigma$ containing $i$ cannot contribute to $c_{i}$, whereas each block $\neq B$ can contribute at most 1 to $c_{i}$. Consequently, we have $c_{i}<k$.

- Since $\sigma$ is $r$-Stirling, the letters $1,2, \ldots, r$ are all minimal in their blocks. In particular, if $1 \leq i \leq r$, the blocks containing $1,2, \ldots, i-1$ cannot contribute to $c_{i}$, so that $c_{i}<k-i+1$.

- Finally, let $S \subseteq[n]$ satisfy $|S|=n-k+1$. We verify $\gamma(S)^{*} \not \leq$ $\left(c_{1}, \ldots, c_{n}\right)$. Working towards a contradiction, suppose $\gamma(S)^{*} \leq\left(c_{1}, \ldots, c_{n}\right)$. Write the reversal $T:=\{n-i+1: i \in S\}$ of $S$ as $T=\left\{t_{1}<\cdots<\right.$ $\left.t_{n-k+1}\right\}$. Since $\sigma$ has $n$ letters and $k$ blocks, at least one element of $T$ must be minimal in its block of $\sigma$. If $t_{n-k+1}$ is minimal in its block of $\sigma$, then

$$
\begin{aligned}
& c_{t_{n-k+1}}=\left|\left\{\begin{array}{lc}
\ell>t_{n-k+1}: & \ell \text { is minimal in its block and } \\
\text { occurs to the right of } t_{n-k+1} \text { in } \sigma
\end{array}\right\}\right| \\
& \leq\left|\left\{t_{n-k+1}+1, \ldots, n-1, n\right\}\right| \\
& =n-t_{n-k+1} \text {. }
\end{aligned}
$$

But the term of $\gamma(S)^{*}$ in position $t_{n-k+1}$ is $n-t_{n-k+1}+1$. We conclude that $t_{n-k+1}$ is not minimal in its block of $\sigma$. If $t_{n-k}$ were minimal in its block of $\sigma$, then

$$
\begin{aligned}
c_{t_{n-k}} & =\left|\left\{\ell>t_{n-k}: \begin{array}{c}
\ell \text { is minimal in its block and } \\
\text { occurs to the right of } t_{n-k} \text { in } \sigma
\end{array}\right\}\right| \\
& \leq\left|\left\{t_{n-k}+1, \ldots, n-1, n\right\}-\left\{t_{n-k+1}\right\}\right| \\
& =n-t_{n-k}-1,
\end{aligned}
$$

But the term of $\gamma(S)^{*}$ in position $t_{n-k}$ is $n-t_{n-k}$. We conclude that $t_{n-k}$ is not minimal in its block of $\sigma$. If $t_{n-k-1}$ were minimal in its 
block of $\sigma$, the same reasoning leads to the contradiction $c_{t_{n-k-1}}<$ $n-t_{n-k-1}-1$, etc. We see that none of the elements in $T$ are minimal in their block of $\sigma$, a contradiction.

In order to show that code $: \mathcal{O P}_{n, k}^{(r)} \rightarrow \mathcal{C}_{n, k}^{(r)}$ is a bijection, we construct its inverse. As this inverse will be defined using an insertion procedure, we denote it $\iota: \mathcal{C}_{n, k}^{(r)} \rightarrow \mathcal{O P}_{n, k}^{(r)}$.

Let $\left(B_{1}|\cdots| B_{k}\right)$ be a sequence of of $k$ possibly empty sets of positive integers. We define the coinversion label of the sets $B_{1}, \ldots, B_{k}$ by labeling the empty sets with $0,1, \ldots, j$ from right to left (where there are $j+1$ empty sets), and then labeling the nonempty sets with $j+1, j+2, \ldots, k-1$ from left to right. An example of coinversion labels is as follows, displayed as superscripts:

$$
\left(\varnothing^{2}\left|13^{3}\right| \varnothing^{1}\left|25^{4}\right| 4^{5} \mid \varnothing^{0}\right) .
$$

By construction, each of the letters $0,1, \ldots, k-1$ appears exactly once as a coinversion label.

Let $\left(c_{1}, \ldots, c_{n}\right) \in \mathcal{C}_{n, k}^{(r)}$. Then $0 \leq c_{i} \leq k-1$ for $1 \leq i \leq n$. We define $\iota\left(c_{1}, \ldots, c_{n}\right)=\left(B_{1}|\cdots| B_{k}\right)$ recursively by starting with the sequence $(\varnothing|\cdots| \varnothing)$ of $k$ copies of the empty set, and for $i=1,2, \ldots, n$ inserting $i$ into the unique block with coinversion label $c_{i}$. Here is an example of this procedure for $(n, k, r)=(9,4,3)$ and $\left(c_{1}, \ldots, c_{9}\right)=(2,0,1,1,1,0,2,1,3)$ :

\begin{tabular}{c|c|c}
$i$ & $c_{i}$ & $\sigma$ \\
\hline 1 & 2 & $\left(\varnothing^{3}\left|\varnothing^{2}\right| \varnothing^{1} \mid \varnothing^{0}\right)$ \\
2 & 0 & $\left(\varnothing^{2}\left|1^{3}\right| \varnothing^{1} \mid \varnothing^{0}\right)$ \\
3 & 1 & $\left(\varnothing^{1}\left|1^{2}\right| \varnothing^{0} \mid 2^{3}\right)$ \\
4 & 1 & $\left(3^{1}\left|1^{2}\right| \varnothing^{0} \mid 2^{3}\right)$ \\
5 & 1 & $\left(34^{1}\left|1^{2}\right| \varnothing^{0} \mid 2^{3}\right)$ \\
6 & 0 & $\left(345^{1}\left|1^{2}\right| \varnothing^{0} \mid 2^{3}\right)$ \\
7 & 2 & $\left(345^{0}\left|1^{1}\right| 6^{2} \mid 2^{3}\right)$ \\
8 & 1 & $\left(345^{0}\left|18^{1}\right| 67^{2} \mid 2^{3}\right)$ \\
9 & 3 & $\left(345^{0}\left|18^{1}\right| 67^{2} \mid 29^{3}\right)$
\end{tabular}

We conclude $\iota(2,0,1,1,1,0,2,1,3)=(345|18| 67 \mid 29)$.

We verify that $\iota$ is a well-defined function $\mathcal{C}_{n, k}^{(r)} \rightarrow \mathcal{O P}_{n, k}^{(r)}$. Let $\left(c_{1}, \ldots, c_{n}\right) \in$ $\mathcal{C}_{n, k}^{(r)}$ and let $\iota\left(c_{1}, \ldots, c_{n}\right)=\left(B_{1}|\cdots| B_{k}\right)=\sigma$. We must show that $1,2, \ldots, r$ lie in distinct blocks of $\sigma$ and that $\sigma$ does not have any empty blocks.

Suppose there exist $1 \leq i<j \leq r$ such that $i$ and $j$ belong to the same block of $\sigma$. Choose the pair $(i, j)$ to be lexicographically minimal with this property and suppose $i, j \in B_{\ell}$. Since the sequence $\left(B_{1}|\cdots| B_{k}\right)$ consists of 
$j-1$ singletons and $k-j+1$ copies of the empty set when $j$ is inserted by $\iota$, the definition of $\iota$ and the fact that $j$ was added to a non-singleton block imply $c_{j} \geq k-j+1$, which contradicts the assumption $\left(c_{1}, \ldots, c_{n}\right) \in \mathcal{C}_{n, k}^{(r)}$. We conclude that $1,2, \ldots, r$ lie in different blocks of $\sigma$.

Now suppose that some of the blocks of $\sigma=\left(B_{1}|\cdots| B_{k}\right)$ are empty. This means that at least $n-k+1$ of the letters in $[n]$ are not minimal in their block of $\sigma$. Let $S$ be the lexicographically first set of $n-k+1$ letters in $[n]$ which are not minimal in their blocks. We will derive the contradiction $\gamma(S)^{*} \leq\left(c_{1}, \ldots, c_{n}\right)$.

Indeed, write the reversal $T=\{n-i+1: i \in S\}$ of $S$ as $T=\left\{t_{1}<\right.$ $\left.\cdots<t_{n-k+1}\right\}$. Let $1 \leq i \leq n-k+1$. By our choice of $S$, we know that the letters in the set difference

$$
\left\{t_{i}+1, t_{i}+2, \ldots, n\right\}-\left\{t_{i+1}, t_{i+2}, \ldots, t_{n-k+1}\right\}
$$

are all minimal in their blocks of $\sigma$; this set has $\left(n-t_{i}\right)-(n-k+1-i)=$ $k-t_{i}+i-1$ elements. Consequently, since $\sigma$ contains at least one empty block, when the $\iota$ inserts $t_{i}$, there are $\geq k-t_{i}+i$ empty blocks. This forces $c_{t_{i}} \geq k-t_{i}+i+1$. Since $k-t_{i}+i+1$ is the term of $\gamma(S)^{*}$ in position $t_{i}$, we conclude $\gamma(S)^{*} \leq\left(c_{1}, \ldots, c_{n}\right)$, which contradicts the assumption that $\left(c_{1}, \ldots, c_{n}\right) \in \mathcal{C}_{n, k}^{(r)}$. Therefore, none of the blocks of $\sigma$ are empty and the function $\iota: \mathcal{C}_{n, k}^{(r)} \rightarrow \mathcal{O P}_{n, k}^{(r)}$ is well-defined. We leave it for the reader to check that code and $\iota$ are mutually inverse.

The code bijection of Theorem 2.2 will have algebraic importance to the theory of Gröbner bases. Recall that a total order $<$ on monomials in $\mathbb{Q}\left[\mathbf{x}_{n}\right]$ is called a monomial order if

- $1 \leq m$ for any monomial $m$, and

- if $m_{1}, m_{2}$, and $m_{3}$ are monomials with $m_{1}<m_{2}$, we have $m_{1} \cdot m_{3}<$ $m_{2} \cdot m_{3}$.

In this paper, we will exclusively use the negative lexicographical term order neglex defined by $x_{1}^{a_{1}} \cdots x_{n}^{a_{n}}<x_{1}^{b_{1}} \cdots x_{n}^{b_{n}}$ if and only if there exists $1 \leq i \leq n$ such that $a_{i}<b_{i}$ and $a_{i+1}=b_{i+1}, \ldots, a_{n}=b_{n}$.

If $<$ is any monomial order and $f \in \mathbb{Q}\left[\mathbf{x}_{n}\right]$ is nonzero, let $\operatorname{in}_{<}(f)$ be the leading term of $f$. Furthermore, if $I \subseteq \mathbb{Q}\left[\mathbf{x}_{n}\right]$ is an ideal, the initial ideal is $\operatorname{in}_{<}(I):=\left\langle\operatorname{in}_{<}(f): f \in I-\{0\}\right\rangle$. A finite subset $G=\left\{g_{1}, \ldots, g_{s}\right\} \subset I$ is called a Gröbner basis if $\operatorname{in}_{<}(I)=\left\langle\operatorname{in}_{<}\left(g_{1}\right), \ldots\right.$, in $\left._{<}\left(g_{s}\right)\right\rangle$. If $G$ is a Gröbner basis for $I$, we necessarily have $I=\langle G\rangle$. Every ideal $I \subseteq \mathbb{Q}\left[\mathbf{x}_{n}\right]$ has a Gröbner basis (with respect to some fixed monomial order $<$ ). 
Let $I \subseteq \mathbb{Q}\left[\mathbf{x}_{n}\right]$ be an ideal and fix a monomial order $<$. If $G=\left\{g_{1}, \ldots, g_{s}\right\}$ is a Gröbner basis for $I$, the set of monomials

$\left\{m: \operatorname{in}_{<}(f) \nmid m\right.$ for all $\left.f \in I-\{0\}\right\}=\left\{m: \operatorname{in}_{<}\left(g_{i}\right) \nmid m\right.$ for $\left.1 \leq i \leq s\right\}$

descends to a $\mathbb{Q}$-vector space basis for $\mathbb{Q}\left[\mathbf{x}_{n}\right] / I$. This is called the standard basis of $\mathbb{Q}\left[\mathbf{x}_{n}\right] / I$. After a monomial order is fixed, any quotient $\mathbb{Q}\left[\mathbf{x}_{n}\right] / I$ has a unique standard basis. The code map precisely describes the standard basis of $R_{n, k}^{(r)}$ in terms of ordered $r$-Stirling partitions.

Theorem 2.3. Let $r \leq k \leq n$ and consider the set of monomials $\mathcal{M}_{n, k}^{(r)}$ given by

$$
\mathcal{M}_{n, k}^{(r)}=\left\{x_{1}^{c_{1}} x_{2}^{c_{2}} \cdots x_{n}^{c_{n}}:\left(c_{1}, c_{2}, \ldots, c_{n}\right)=\operatorname{code}(\sigma) \text { for some } \sigma \in \mathcal{O P}_{n, k}^{(r)}\right\} .
$$

1. The set $\mathcal{M}_{n, k}^{(r)}$ is the standard basis for the $\mathbb{Q}$-vector space $R_{n, k}^{(r)}$ with respect to the neglex monomial order.

2. The set $\mathcal{M}_{n, k}^{(r)}$ is a $\mathbb{Z}$-basis for the $\mathbb{Z}$-module $S_{n, k}^{(r)}$.

Proof. 1. We begin by proving the inequality $\operatorname{dim}\left(R_{n, k}^{(r)}\right) \geq\left|\mathcal{O P}_{n, k}^{(r)}\right|$. Consider $k$ distinct rational numbers $\alpha_{1}, \ldots, \alpha_{k}$ and let $Y_{n, k}^{(r)} \subset \mathbb{Q}^{n}$ be the family of points $\left(y_{1}, \ldots, y_{n}\right)$ such that

- $\left\{y_{1}, \ldots, y_{n}\right\}=\left\{\alpha_{1}, \ldots, \alpha_{k}\right\}$, and

- the coordinates $y_{1}, \ldots, y_{r}$ are distinct.

It is evident that $Y_{n, k}^{(r)}$ carries an action of the symmetric group product $S_{r} \times S_{n-r}$, and that this affords an identification of $Y_{n, k}^{(r)}$ with $\mathcal{O} \mathcal{P}_{n, k}^{(r)}$.

Let $\mathbf{I}\left(Y_{n, k}^{(r)}\right) \subseteq \mathbb{Q}\left[\mathbf{x}_{n}\right]$ be the ideal of polynomials in $\mathbb{Q}\left[\mathbf{x}_{n}\right]$ which vanish on $Y_{n, k}^{(r)}$. We have

$$
\mathbb{Q}\left[\mathbf{x}_{n}\right] / \mathbf{I}\left(Y_{n, k}^{(r)}\right) \cong \mathbb{Q}\left[Y_{n, k}^{(r)}\right] \cong \mathbb{Q}\left[\mathcal{O P}_{n, k}^{(r)}\right]
$$

as $S_{r} \times S_{n-r}$-modules. If $f \in \mathbf{I}\left(Y_{n, k}^{(r)}\right)$ is nonzero, let $\tau(f)$ denote the homogeneous component of $f$ of highest degree and set

$$
\mathbf{T}\left(Y_{n, k}^{(r)}\right):=\left\langle\tau(f): f \in \mathbf{I}\left(Y_{n, k}^{(r)}\right)-\{0\}\right\rangle
$$


We have the further $S_{r} \times S_{n-r}$-module isomorphism

$$
\mathbb{Q}\left[\mathbf{x}_{n}\right] / \mathbf{T}\left(Y_{n, k}^{(r)}\right) \cong \mathbb{Q}\left[\mathbf{x}_{n}\right] / \mathbf{I}\left(Y_{n, k}^{(r)}\right) \cong \mathbb{Q}\left[Y_{n, k}^{(r)}\right] \cong \mathbb{Q}\left[\mathcal{O} \mathcal{P}_{n, k}^{(r)}\right]
$$

Proving the dimension inequality $\operatorname{dim}\left(R_{n, k}^{(r)}\right) \geq\left|\mathcal{O} \mathcal{P}_{n, k}^{(r)}\right|$ therefore reduces to showing the containment $I_{n, k}^{(r)} \subseteq \mathbf{T}\left(Y_{n, k}^{(r)}\right)$; we do this by considering the generators of $I_{n, k}^{(r)}$.

- Let $1 \leq i \leq n$; we show that the monomial $x_{i}^{k}$ lies in $\mathbf{T}\left(Y_{n, k}^{(r)}\right)$. This follows from the fact that $\left(x_{i}-\alpha_{1}\right)\left(x_{i}-\alpha_{2}\right) \cdots\left(x_{i}-\alpha_{k}\right) \in \mathbf{I}\left(Y_{n, k}^{(r)}\right)$.

- We show that $e_{n}\left(\mathbf{x}_{n}\right), e_{n-1}\left(\mathbf{x}_{n}\right), \ldots, e_{n-k+1}\left(\mathbf{x}_{n}\right) \in \mathbf{T}\left(Y_{n, k}^{(r)}\right)$. Indeed, introduce a new variable $t$ and consider the rational function

$$
\frac{\left(1-x_{1} t\right) \cdots\left(1-x_{n} t\right)}{\left(1-\alpha_{1} t\right) \cdots\left(1-\alpha_{k} t\right)}=\sum_{i, j}(-1)^{i} e_{i}\left(\mathbf{x}_{n}\right) h_{j}\left(\alpha_{1}, \ldots, \alpha_{k}\right) \cdot t^{i+j}
$$

If $\left(x_{1}, \ldots, x_{n}\right) \in Y_{n, k}^{(r)}$ the factors of the denominator cancel with $k$ factors in the numerator, yielding a polynomial in $t$ of degree $n-k$. If $n-k+1 \leq i \leq n$, taking the coefficient of $t^{i}$ on both sides leads to $e_{i}\left(\mathbf{x}_{n}\right) \in \mathbf{T}\left(Y_{n, k}^{(r)}\right)$.

$$
\frac{\left(1-\alpha_{1} t\right) \cdots\left(1-\alpha_{k} t\right)}{\left(1-x_{1} t\right) \cdots\left(1-x_{r} t\right)}=\sum_{i, j}(-1)^{i} e_{i}\left(\alpha_{1}, \ldots, \alpha_{k}\right) h_{j}\left(\mathbf{x}_{r}\right) \cdot t^{i+j}
$$

If $\left(x_{1}, \ldots, x_{n}\right) \in Y_{n, k}^{(r)}$, the factors in the denominator cancel with $r$ factors in the numerator, yielding a polynomial in $t$ of degree $k-r$. If $k-r+1 \leq j \leq k$, taking the coefficient of $t^{i}$ on both sides leads to $h_{j}\left(\mathbf{x}_{r}\right) \in \mathbf{T}\left(Y_{n, k}^{(r)}\right)$.

This completes the proof that $\operatorname{dim}\left(R_{n, k}^{(r)}\right) \geq\left|\mathcal{O P}_{n, k}^{(r)}\right|$.

Given any subset $S \subseteq[n]$ with reverse skip composition $\gamma(S)^{*}=\left(a_{1}, \ldots, a_{n}\right)$, let $\mathbf{x}(S)^{*}:=x_{1}^{a_{1}} \cdots x_{n}^{a_{n}}$ be the associated reverse skip monomial. By [3, Sec. 3], we have $\mathbf{x}(S)^{*} \in \operatorname{in}_{<}\left(I_{n, k}^{(r)}\right)$ whenever $S \subseteq[n]$ satisfies $|S|=n-k+1$. Furthermore, the identities

$$
h_{d}\left(x_{1}, \ldots, x_{i-1}, x_{i}\right)-x_{i} h_{d-1}\left(x_{1}, \ldots, x_{i-1}, x_{i}\right)=h_{d}\left(x_{1}, \ldots, x_{i-1}\right)
$$


imply that $x_{1}^{k}, x_{2}^{k-1}, \ldots, x_{r}^{k-r-1} \in \operatorname{in}_{<}\left(I_{n, k}^{(r)}\right)$. Finally, we have $x_{r+1}^{k}, \ldots, x_{n-1}^{k}, x_{n}^{k} \in$ $\operatorname{in}_{<}\left(I_{n, k}^{(r)}\right)$. Theorem 2.2 implies that the monomials in $\mathcal{M}_{n, k}^{(r)}$ are precisely those monomials in $\mathbb{Q}\left[\mathbf{x}_{n}\right]$ which are not divisible by any of the three classes of elements of $\operatorname{in}_{<}\left(I_{n, k}^{(r)}\right)$ listed above. Again by Theorem 2.2 we have $\operatorname{dim}\left(R_{n, k}^{(r)}\right) \geq$ $\left|\mathcal{O P}_{n, k}^{(r)}\right|=\left|\mathcal{M}_{n, k}^{(r)}\right|$, so that $\mathcal{M}_{n, k}^{(r)}$ is the standard basis of $R_{n, k}^{(r)}$.

2. From Item 1 of this theorem, we know that the set $\mathcal{M}_{n, k}^{(r)}$ descends to a linearly independent subset of $S_{n, k}^{(r)}$; we need only show that $\mathcal{M}_{n, k}^{(r)}$ descends to a $\mathbb{Z}$-spanning set of $S_{n, k}^{(r)}$. To this end, let $m$ be any monomial in $\mathbb{Z}\left[\mathbf{x}_{n}\right]$. We show inductively that $m+J_{n, k}^{(r)}$ lies in the $\mathbb{Z}$-span of $\mathcal{M}_{n, k}^{(r)}$. If $m \in \mathcal{M}_{n, k}^{(r)}$ this is obvious. Otherwise, one of the following three things must be true:

1. There exists $1 \leq i \leq r$ such that $x_{i}^{k-i+1} \mid m$.

2. There exists $r+1 \leq i \leq n$ such that $x_{i}^{k} \mid m$.

3. There exists $S \subseteq[n]$ with $|S|=n-k+1$ such that $\mathbf{x}(S)^{*} \mid m$.

If (1) holds, Equation (25) implies $h_{k-i+1}\left(x_{1}, x_{2}, \ldots, x_{i}\right) \in J_{n, k}^{(r)}$. As a consequence, we have

$x_{i}^{k-i+1} \equiv$ a $\mathbb{Z}$-linear combination of monomials $<x_{i}^{k-i+1}$ in neglex $\left(\bmod J_{n, k}^{(r)}\right)$.

If we multiply through by the monomial $m / x_{i}^{k-i+1}$, we see that

$$
m \equiv \text { a } \mathbb{Z} \text {-linear combination of monomials }<m \text { in neglex }\left(\bmod J_{n, k}^{(r)}\right),
$$

so that inductively we see that $m+J_{n, k}^{(r)}$ lies in the span of $\mathcal{M}_{n, k}^{(r)}$.

If (2) holds, then $m \in J_{n, k}^{(r)}$, so certainly $m+J_{n, k}^{(r)}=0$ lies in the $\mathbb{Z}$-span of $\mathcal{M}_{n, k}^{(r)}$.

If (3) holds, let $\kappa_{\gamma(S)^{*}}\left(\mathbf{x}_{n}\right) \in \mathbb{Z}\left[\mathbf{x}_{n}\right]$ be the Demazure character attached to the reverse skip composition $\gamma(S)^{*}$. This is a certain polynomial in the variables $x_{1}, \ldots, x_{n}$ with nonnegative integer coefficients. The precise form of this polynomial is not important for us, but we have (see e.g. [3, Lem.

$\kappa_{\gamma(S)^{*}}\left(\mathbf{x}_{n}\right)=\mathbf{x}(S)^{*}+$ a $\mathbb{Z}$-linear combination of terms $<\mathbf{x}(S)^{*}$ in neglex.

By [3, Lem 3.4] we have $\kappa_{\gamma(S)^{*}}\left(\mathbf{x}_{n}\right) \in J_{n, k}^{(r)}$, so that Equation (28) implies $(29)$

$$
\mathbf{x}(S)^{*} \equiv \text { a } \mathbb{Z} \text {-linear combination of terms }<\mathbf{x}(S)^{*} \text { in neglex }\left(\bmod J_{n, k}^{(r)}\right) \text {. }
$$


If we multiply Equation (29) through by the monomial $m / \mathbf{x}(S)^{*}$, we get

$$
m \equiv \text { a } \mathbb{Z} \text {-linear combination of terms }<m \text { in neglex }\left(\bmod J_{n, k}^{(r)}\right) \text {. }
$$

so that inductively we see that $m+J_{n, k}^{(r)}$ lies in the $\mathbb{Z}$-span of $\mathcal{M}_{n, k}^{(r)}$.

When $r=0$, Theorem 2.3 is equivalent to a result of Haglund, Rhoades, and Shimozono [3, Thm. 4.13]. However, the proof of Theorem 2.3 is much more direct that of [3, Thm. 4.13] (and those in [3, Sec. 4] in general); whereas we associate an explicit standard basis element $x_{1}^{c_{1}} \cdots x_{n}^{c_{n}}$ to any ordered set partition $\sigma$, the description of the standard bases in [3] is recursive in nature. We exhibit this link between ordered set partitions and standard basis elements with an example.

Example 2.4. To illustrate Theorem 2.3, we give the standard basis of $R_{4,3}^{(2)}$ with respect to neglex.

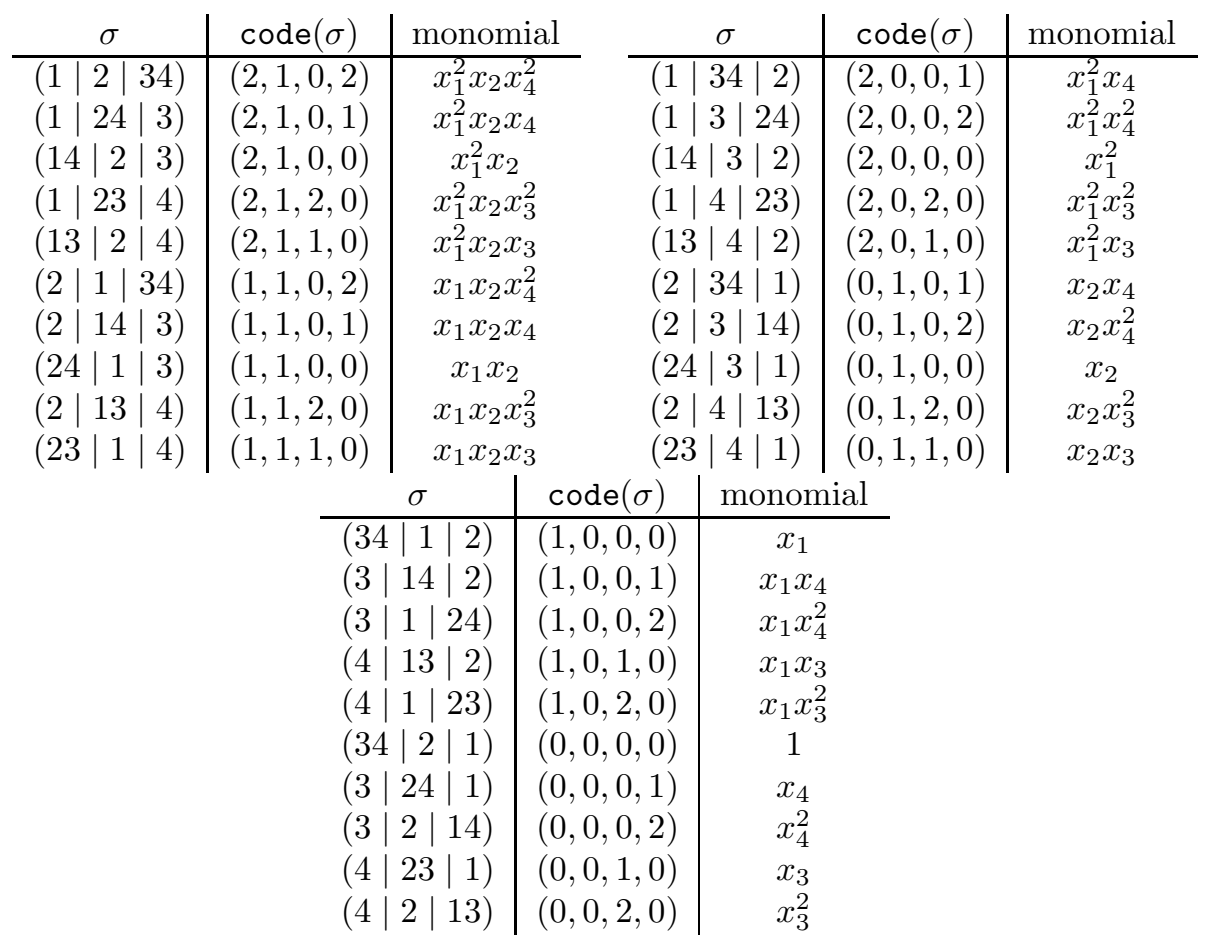

As an application of Theorem 2.3, we can describe the Hilbert series of $R_{n, k}^{(r)}$ in terms of the coinv statistic. 
Corollary 2.5. The Hilbert series of $R_{n, k}^{(r)}$ is given by

$$
\operatorname{Hilb}\left(R_{n, k}^{(r)} ; q\right)=\sum_{\sigma \in \mathcal{O P}_{n, k}^{(r)}} q^{\operatorname{coinv}(\sigma)}
$$

As another application of Theorem 2.3, we can describe the ungraded isomorphism type of $R_{n, k}^{(r)}$ as a module over $S_{r} \times S_{n-r}$. When $r=k=n$, this is Chevalley's classical result [1] that the coinvariant ring is isomorphic to the regular representation of $S_{n}$.

Corollary 2.6. We have an isomorphism of ungraded $S_{r} \times S_{n-r}$-modules

$$
R_{n, k}^{(r)} \cong \mathbb{Q}\left[\mathcal{O P}_{n, k}^{(r)}\right]
$$

It seems that the isomorphism type of $R_{n, k}^{(r)}$ as a graded $S_{r} \times S_{n-r}$-module can be described in terms of known graded modules by the (graded) tensor product decomposition

$$
R_{n, k}^{(r)} \cong R_{r} \otimes_{\mathbb{C}} \varepsilon_{r} R_{n, k}
$$

In the conjectural isomorphism (33) of graded $S_{r} \times S_{n-r}$-modules,

- $R_{r}=\mathbb{Q}\left[\mathbf{x}_{r}\right] /\left\langle e_{1}\left(\mathbf{x}_{r}\right), \ldots, e_{r}\left(\mathbf{x}_{r}\right)\right\rangle$ is the classical coinvariant ring in the first $r$ variables $\mathbf{x}_{r}$, with its graded action of $S_{r}$,

- $R_{n, k}=R_{n, k}^{(0)}$ is the graded $S_{n}$-module $\mathbb{Q}\left[\mathbf{x}_{n}\right] /\left\langle x_{1}^{k}, \ldots, x_{n}^{k}, e_{n}\left(\mathbf{x}_{n}\right), \ldots, e_{n-k+1}\left(\mathbf{x}_{n}\right)\right\rangle$, and

- $\varepsilon_{r} \in \mathbb{Q}\left[S_{n}\right]$ is the group algebra element

$$
\varepsilon_{r}:=\sum_{w \in S_{r}} \operatorname{sign}(w) \cdot w
$$

which antisymmetrizes over the subgroup $S_{r} \subseteq S_{n}$ (acting on the first $r$ letters), so that $S_{n-r}$ (acting on the last $n-r$ letters) commutes with $\varepsilon_{r}$ and therefore

- $\varepsilon_{r} R_{n, k}$ is naturally a $S_{n-r}$-module, and

- the action of the product group $S_{r} \times S_{n-r}$ on the tensor product is given by

$$
\left(w_{1} \times w_{2}\right) \cdot\left(v_{1} \otimes v_{2}\right):=\left(w_{1} \cdot v_{1}\right) \otimes\left(w_{2} \cdot v_{2}\right)
$$




\section{Line configurations and $r$-Stirling partitions}

We shift focus from algebra to geometry and initiate the study of $X_{n, k}^{(r)}$. In order to study the variety $X_{n, k}^{(r)}$, we will need to break it into pieces in a reasonable way. For this we will use the notion of an affine paving (called a cellular decomposition in [5]).

Let $X$ be a smooth irreducible complex algebraic variety. An affine paving of $X$ is an ordered partition

$$
X=C_{1} \sqcup \cdots \sqcup C_{m}
$$

such that

- for all $i$, the union $C_{1} \sqcup \cdots \sqcup C_{i}$ is a closed subvariety of $X$, and

- $C_{i}$ is isomorphic as a variety to the affine space $\mathbb{C}^{n_{i}}$, for some integer $n_{i}$.

The $C_{i}$ are referred to as the cells of the affine paving and we will say that the partition $\left\{C_{1}, \ldots, C_{m}\right\}$ induces an affine paving of $X$. In this situation, the classes of the cell closures $\left\{\left[\overline{C_{1}}\right], \ldots,\left[\overline{C_{m}}\right]\right\}$ give a $\mathbb{Z}$-basis for the (singular) cohomology ring $H^{\bullet}(X)$.

The projective space $\mathbb{P}^{k-1}$ has an affine paving induced by the cells $\left\{C_{1}, C_{2}, \ldots, C_{k}\right\}$, where

$$
C_{i}=\left\{\left[x_{1}: x_{2}: \cdots: x_{k}\right] \in \mathbb{P}^{k-1}: x_{1}=\cdots=x_{i-1}=0 \text { and } x_{i} \neq 0\right\} .
$$

Taking products of these cells gives the standard affine paving of $\left(\mathbb{P}^{k-1}\right)^{n}$ whose cells are indexed by words $w=w_{1} \ldots w_{n} \in[k]^{n}$. Following [5], we will consider a different affine paving of $\left(\mathbb{P}^{k-1}\right)^{n}$ whose cells are again indexed by words in $[k]^{n}$. In order to describe this paving, we will need some terminology.

Let $\mathrm{Mat}_{k \times n}$ stand for the affine space of all complex $k \times n$ matrices $m$. Let $\mathcal{U}_{n, k}^{(r)}$ be the Zariski open subset

$$
\mathcal{U}_{n, k}^{(r)}:=\left\{m \in \operatorname{Mat}_{k \times n}: \begin{array}{c}
\text { the matrix } m \text { has full rank, no zero } \\
\text { columns, and the first } r \text { columns } \\
\text { of } m \text { are linearly independent }
\end{array}\right\} .
$$

If we let $T \subset G L_{n}$ be the rank $n$ diagonal torus, then $T$ acts freely on the columns of $\mathcal{U}_{n, k}^{(r)}$ and we may identify the orbit space as $\mathcal{U}_{n, k}^{(r)} / T=X_{n, k}^{(r)}$. Furthermore, we consider the larger Zariski open set $\mathcal{V}_{n, k}$ given by

$$
\mathcal{V}_{n, k}:=\left\{m \in \operatorname{Mat}_{k \times n}: m \text { has no zero columns }\right\} .
$$


This time we have the identification $\mathcal{V}_{n, k} / T=\left(\mathbb{P}^{k-1}\right)^{n}$.

Let $w=w_{1} \ldots w_{n} \in[k]^{n}$ be a word in the letters $1,2, \ldots, k$ of length $n$. An index $1 \leq j \leq n$ is called initial if $w_{j}$ is the first occurrence of its letter in $w$; let $\operatorname{in}(w)=\left\{j_{1}<j_{2}<\cdots<j_{s}\right\}$ be the set of initial indices in $w$. For example, if $w=242141 \in[4]^{6}$ then $\operatorname{in}(w)=\{1,2,4\}$. The $k \times n$ pattern matrix $\operatorname{PM}(w)$ has entries in the set $\{0,1, \star\}$ as follows:

$$
\operatorname{PM}(w)_{i, j}= \begin{cases}1 & \text { if } w_{j}=i \\ 0 & \text { if the letter } i \text { does not appear in } w \\ \star & \text { if } j \in \operatorname{in}(w), i<w_{j}, \text { and there exists } j^{\prime}<j \text { such that } w_{j^{\prime}}=i \\ 0 & \text { if } j \in \operatorname{in}(w) \text { and }\left(i>w_{j} \text { or there does not exist } j^{\prime}<j \text { such that } w_{j^{\prime}}=i\right) \\ \star & \text { if } j \notin \operatorname{in}(w), i \neq w_{j}, \text { and the first } i \text { appears before the first } w_{j} \text { in } w \\ 0 & \text { if } j \notin \operatorname{in}(w), i \neq w_{j}, \text { and the first } i \text { appears after the first } w_{j} \text { in } w .\end{cases}
$$

In our example,

$$
\operatorname{PM}(242141)=\left(\begin{array}{cccccc}
0 & 0 & 0 & 1 & 0 & 1 \\
1 & \star & 1 & 0 & \star & \star \\
0 & 0 & 0 & 0 & 0 & 0 \\
0 & 1 & 0 & 0 & 1 & \star
\end{array}\right)
$$

For any word $w=w_{1} \ldots w_{n} \in[k]^{n}$, let $\widehat{C_{w}}$ be the affine space of all matrices obtained by replacing the $\star$ 's in $\operatorname{PM}(w)$ by complex numbers. Let $U \subset G L_{k}(\mathbb{C})$ be the unipotent subgroup of lower triangular matrices with 1 's on the diagonal. We define a subset $C_{w} \subseteq\left(\mathbb{P}^{k-1}\right)^{n}$ by

$$
C_{w}:=\text { image of } U \cdot \widehat{C_{w}} \text { in }\left(\mathbb{P}^{k-1}\right)^{n} \text {. }
$$

It follows from [5] that $C_{w}$ is isomorphic as a variety to an affine space.

Proposition 3.1. ([5]) For any $k \leq n$, the set $\left\{C_{w}: w \in[k]^{n}\right\}$ induces an affine paving of $\left(\mathbb{P}^{k-1}\right)^{n}$.

The affine paving of Proposition 3.1 induces an affine paving of $X_{n, k}^{(r)}$. To describe this paving, we define $\mathcal{W}_{n, k}^{(r)}$ to be the family of words $w=$ $w_{1} w_{2} \ldots w_{n} \in[k]^{n}$ such that the letters $1,2, \ldots, k$ all appear in $w$ and that the first $r$ letters $w_{1}, w_{2}, \ldots, w_{r}$ of $w$ are distinct.

Proposition 3.2. The family of cells $\left\{C_{w}: w \in \mathcal{W}_{n, k}^{(r)}\right\}$ induces an affine paving of the variety $X_{n, k}^{(r)}$. 
Proof. Let $w \in[k]^{n}$ be any word and consider the cell $C_{w} \subset\left(\mathbb{P}^{k-1}\right)^{n}$. The definition of the pattern matrix $\operatorname{PM}(w)$ implies that $C_{w} \subset X_{n, k}^{(r)}$ if $w \in \mathcal{W}_{n, k}^{(r)}$ and $C_{w} \cap X_{n, k}^{(r)}=\varnothing$ otherwise. Now observe that the total order on the cells $\left\{C_{w}: w \in[k]^{n}\right\}$ inducing the affine paving of Proposition 3.1 may be taken to start with those $w \notin \mathcal{W}_{n, k}^{(r)}$ (in some order) and end with those $w \in \mathcal{W}_{n, k}^{(r)}$ (in some order). The claim follows.

Our next task is to present the cohomology of $X_{n, k}^{(r)}$ as the quotient $S_{n, k}^{(r)}$ and describe the images of the $\mathbb{Z}$-basis $\left\{\left[\overline{C_{w}}\right]: w \in \mathcal{W}_{n, k}^{(r)}\right\}$ afforded by Proposition 3.2. We being by recalling the standard presentation of the cohomology of $\left(\mathbb{P}^{k-1}\right)^{n}$.

The cohomology of $\left(\mathbb{P}^{k-1}\right)^{n}$ is presented as

$$
H^{\bullet}\left(\left(\mathbb{P}^{k-1}\right)^{n}\right)=\mathbb{Z}\left[\mathbf{x}_{n}\right] /\left\langle x_{1}^{k}, \ldots, x_{n}^{k}\right\rangle,
$$

where $x_{i}$ represents the Chern class $c_{1}\left(\ell_{i}^{*}\right) \in H^{2}\left(\left(\mathbb{P}^{k-1}\right)^{n}\right)$ of the dual to the $i^{\text {th }}$ tautological line bundle $\ell_{i} \rightarrow\left(\mathbb{P}^{k-1}\right)^{n}$.

Given a word $w \in[k]^{n}$, a polynomial representative for $\left[\overline{C_{w}}\right] \in H^{\bullet}\left(\left(\mathbb{P}^{k-1}\right)^{n}\right)$ was calculated in [5]. In order to state it, we recall the classical Schubert polynomials attached to permutations in $S_{n}$.

The Schubert polynomials $\left\{\mathfrak{S}_{w}: w \in S_{n}\right\}$ are defined recursively by

$$
\begin{cases}\mathfrak{S}_{w_{0}}=x_{1}^{n-1} x_{2}^{n-2} \cdots x_{n}^{0} & \text { for } w_{0}=n(n-1) \ldots 1 \\ \mathfrak{S}_{w s_{i}}=\partial_{i} \mathfrak{S}_{w} & \text { if } w_{i}>w_{i+1}\end{cases}
$$

Here $w s_{i}$ is the permutation whose one-line notation $w s_{i}=w_{1} \ldots w_{i+1} w_{i} \ldots w_{n}$ is obtained from that of $w$ by interchanging the letters in positions $i$ and $i+1$ and $\partial_{i}$ is the divided difference operator

$$
\partial_{i}\left(f\left(x_{1}, \ldots, x_{n}\right)\right)=\frac{f\left(x_{1}, \ldots, x_{i}, x_{i+1}, \ldots, x_{n}\right)-f\left(x_{1}, \ldots, x_{i+1}, x_{i}, \ldots, x_{n}\right)}{x_{i}-x_{i+1}} .
$$

In order to extend Schubert polynomials from permutations in $S_{n}$ to words in $[k]^{n}$, we will need some notation. A word $w$ is called convex if it does not have a subword of the form $\ldots i \ldots j \ldots i \ldots$ Any word $w$ has a unique convexification $\operatorname{conv}(w)$ which is characterized by being convex, having the same letter multiplicities as $w$, and having its initial letters appear in the same order from left to right. For example, we have $\operatorname{conv}(242141)=224411$. Furthermore, let $\sigma(w) \in S_{n}$ be the unique permutation with a minimal 
number of inversions which sorts $w$ to $\operatorname{conv}(w)$; in our example $\sigma(242141)=$ $132546 \in S_{6}$.

Suppose $w=w_{1} \ldots w_{n} \in[k]^{n}$ is a convex word with $m$ distinct letters. Let $\left\{i_{1}<i_{2}<\cdots<i_{k-m}\right\}$ be the letters in $[k]$ which do not appear in $w$. We define the standardization $\operatorname{st}(w)=\operatorname{st}(w)_{1} \ldots \operatorname{st}(w)_{n+k-m} \in S_{n+k-m}$ to be the permutation obtained from $w$ by fixing the initial letters of $w$, replacing the non-initial letters of $w$ from left to right with $k+1, k+2, \ldots, n+k-m$, and appending the sequence $i_{1} i_{2} \ldots i_{k-m}$ to the end. For example, if $(n, k)=$ $(7,5)$ and $w=3344411$ then $\operatorname{st}(w)=364781925 \in S_{9}$.

Let $w \in[k]^{n}$ be an arbitrary word of length $n$ in the letters $1,2, \ldots, k$. The word Schubert polynomial $\mathfrak{S}_{w}$ is defined by

$$
\mathfrak{S}_{w}:=\sigma(w)^{-1} \cdot \mathfrak{S}_{\mathrm{st}(\operatorname{conv}(w))} \cdot
$$

Although the permutation st $(\operatorname{conv}(w))$ will lie in a symmetric group of rank $>n$ when $w$ does not contain all of the letters $1,2, \ldots, k$, the polynomial $\mathfrak{S}_{w}$ depends only on the variables $x_{1}, x_{2}, \ldots, x_{n}$ so that $\mathfrak{S}_{w} \in \mathbb{Z}\left[\mathbf{x}_{n}\right]$. Pawlowski and Rhoades proved [5] that the closure of the cell $C_{w}$ is represented by $\mathfrak{S}_{w}$ under the presentation (42):

$$
\left[\overline{C_{w}}\right] \text { is represented by } \mathfrak{S}_{w} \text { in } H^{\bullet}\left(\left(\mathbb{P}^{k-1}\right)^{n}\right) .
$$

Theorem 3.3. Let $r \leq k \leq n$. The singular cohomology of $X_{n, k}^{(r)}$ may be presented as

$$
H^{\bullet}\left(X_{n, k}^{(r)}\right)=S_{n, k}^{(r)}
$$

Furthermore, under the presentation (47), if $w \in \mathcal{W}_{n, k}^{(r)}$ the cell closure $\overline{C_{w}}$ is represented in $H^{\bullet}\left(X_{n, k}^{(r)}\right)$ by $\mathfrak{S}_{w}$.

Proof. Consider the affine paving $\left\{C_{w}: w \in[k]^{n}\right\}$ of $\left(\mathbb{P}^{k-1}\right)^{n}$ afforded by Proposition 3.1. If $w \notin \mathcal{W}_{n, k}^{(r)}$, we have $\overline{C_{w}} \cap X_{n, k}^{(r)}=\varnothing$. By Proposition 3.2, it follows that $X_{n, k}^{(r)}$ is obtained from $\left(\mathbb{P}^{k-1}\right)^{n}$ by excising the union of cell closures $\bigcup_{w \in[k]^{n}-\mathcal{W}_{n, k}^{(r)}} \overline{C_{w}}$. It follows (see [5]) that the cohomology ring $H^{\bullet}\left(X_{n, k}^{(r)}\right)$ may be presented as

$$
H^{\bullet}\left(X_{n, k}^{(r)}\right)=H^{\bullet}\left(\left(\mathbb{P}^{k-1}\right)^{n}\right) / J
$$

where $J \subseteq H^{\bullet}\left(\left(\mathbb{P}^{k-1}\right)^{n}\right)$ is the ideal generated by those $\left[\overline{C_{w}}\right]$ for which $w \in[k]^{n}-\mathcal{W}_{n, k}^{(r)}$. If we use the presentation of $H^{\bullet}\left(\left(\mathbb{P}^{k-1}\right)^{n}\right)$ given in (42) 
together with the polynomial representatives (46), we can write

$$
H^{\bullet}\left(X_{n, k}^{(r)}\right)=\mathbb{Z}\left[\mathbf{x}_{n}\right] / I,
$$

where $I \subseteq \mathbb{Z}\left[\mathbf{x}_{n}\right]$ is the ideal generated by $x_{1}^{k}, x_{2}^{k}, \ldots, x_{n}^{k}$ together with $\left\{\mathfrak{S}_{w}\right.$ : $\left.w \in[k]^{n}-\mathcal{W}_{n, k}^{(r)}\right\}$.

Claim: We have $J_{n, k}^{(r)} \subseteq I$.

To prove the Claim, we show that every generator of $J_{n, k}^{(r)}$ lies in $I$. We handle each type of generator separately.

- The generators $x_{1}^{k}, x_{2}^{k}, \ldots, x_{n}^{k}$ of $J_{n, k}^{(r)}$ are also generators of $I$.

- For the generators $e_{n-i+1}\left(\mathbf{x}_{n}\right)$ (where $1 \leq i \leq k$ ) of $J_{n, k}^{(r)}$ we do the following. For $1 \leq i \leq k$ let $w^{i}$ be the unique weakly increasing word in $[k]^{n}$ containing exactly the letters $[k]-\{i\}$ and whose first $k-1$ letters are distinct. For example, the word $w^{3} \in[5]^{7}$ is $w^{3}=1245555$. Since $i$ does not appear in $w^{i}$, we have $w^{i} \notin \mathcal{W}_{n, k}^{(r)}$, so that $\mathfrak{S}_{w^{i}}$ is a generator of $I$. Furthermore, we have

$$
\operatorname{st}\left(\operatorname{conv}\left(w^{i}\right)\right)=12 \ldots(i-1)(i+1) \ldots n(n+1) i \in S_{n+1}
$$

which implies $\mathfrak{S}_{w^{i}}=e_{n-i+1}\left(\mathbf{x}_{n}\right)$.

- Finally, we consider the generators $h_{k-i+1}\left(\mathbf{x}_{r}\right)$ (where $1 \leq i \leq r$ ) of $J_{n, k}^{(r)}$. These generators are not in general generators of $I$, but we show that they nevertheless are contained in $I$. If $k=n$ then $X_{n, k}^{(r)}=X_{n, n}$ so that the theorem follows from [5]; we assume that $k<n$.

For $1 \leq i \leq r-1$, let $v^{i} \in[k]^{n}$ be the following weakly increasing word:

$$
v^{i}=12 \ldots(i-1) i i(i+1)(i+2) \ldots(k-1) k \ldots k .
$$

For example, the word $v^{3} \in[5]^{7}$ is $v^{3}=12334555$. Since $k<n$, every letter in $[k]$ appears in $v^{i}$. However, since the first $r$ letters of $v^{i}$ are not distinct, we have $v^{i} \notin \mathcal{W}_{n, k}^{(r)}$, so that $\mathfrak{S}_{v^{i}}$ is a generator of $I$. We have

$$
\operatorname{st}\left(\operatorname{conv}\left(v^{i}\right)\right)=12 \ldots(i-1) i(k+1)(i+1)(i+2) \ldots n \in S_{n}
$$

which implies $\mathfrak{S}_{v^{i}}=h_{k-i}\left(\mathbf{x}_{i+1}\right)$.

The above paragraph shows that

$$
h_{k-r+1}\left(\mathbf{x}_{r}\right), h_{k-r+2}\left(\mathbf{x}_{r-1}\right), \ldots, h_{k-1}\left(\mathbf{x}_{2}\right) \in I .
$$


The variable power $h_{k}\left(\mathbf{x}_{1}\right)=x_{1}^{k}$ also lies in $I$. The identity (50)

$$
h_{d}\left(x_{1}, \ldots, x_{i-1}, x_{i}\right)=x_{i} \cdot h_{d-1}\left(x_{1}, \ldots, x_{i-1}, x_{i}\right)+h_{d}\left(x_{1}, \ldots, x_{i-1}\right)
$$

together with the fact that $I$ is an ideal in $\mathbb{Z}\left[\mathbf{x}_{n}\right]$ can be used to show that

$$
h_{k-r+1}\left(\mathbf{x}_{r}\right), h_{k-r+2}\left(\mathbf{x}_{r}\right), \ldots, h_{k}\left(\mathbf{x}_{r}\right) \in I,
$$

which is what we wanted to show. This completes the proof of the Claim.

By our Claim, we have a canonical surjection of $\mathbb{Z}$-modules

$$
S_{n, k}^{(r)}=\mathbb{Z}\left[\mathbf{x}_{n}\right] / J_{n, k}^{(r)} \rightarrow \mathbb{Z}\left[\mathbf{x}_{n}\right] / I=H^{\bullet}\left(X_{n, k}^{(r)}\right) .
$$

By Theorem 2.3, the module $S_{n, k}^{(r)}$ is a free $\mathbb{Z}$-module of rank $\left|\mathcal{O} \mathcal{P}_{n, k}^{(r)}\right|$. By Proposition 3.2 , the cohomology ring $H^{\bullet}\left(X_{n, k}^{(r)}\right)$ is a free $\mathbb{Z}$-module of rank $\left|\mathcal{W}_{n, k}^{(r)}\right|$. Since we have $\left|\mathcal{O} \mathcal{P}_{n, k}^{(r)}\right|=\left|\mathcal{W}_{n, k}^{(r)}\right|$ and any surjection between free $\mathbb{Z}$-modules of the same rank must be an isomorphism, we obtain the presentation (47) of the cohomology of $X_{n, k}^{(r)}$. The last sentence of the theorem follows from (46).

The cohomology representatives of the cell closures in any affine paving of a smooth irreducible variety $X$ give rise to a $\mathbb{Z}$-basis for the cohomology ring $H^{\bullet}(X)$. Theorem 3.3 therefore yields the following immediate corollary.

Corollary 3.4. Let $r \leq k \leq n$. The set of polynomials $\left\{\mathfrak{S}_{w}: w \in \mathcal{W}_{n, k}^{(r)}\right\}$ descends to a $\mathbb{Z}$-basis for $S_{n, k}^{(r)}$.

We have the following isomorphisms of ungraded $S_{r} \times S_{n-r}$-modules:

$$
H^{\bullet}\left(X_{n, k}^{(r)} ; \mathbb{Q}\right) \cong \mathbb{Q} \otimes_{\mathbb{Z}} H^{\bullet}\left(X_{n, k}^{(r)}\right) \cong \mathbb{Q} \otimes_{\mathbb{Z}} S_{n, k}^{(r)} \cong R_{n, k}^{(r)} \cong \mathbb{Q}\left[\mathcal{O} \mathcal{P}_{n, k}^{(r)}\right]
$$

The first of these isomorphisms follows from the Universal Coefficient Theorem (see e.g. [4]) and the fact that $H^{\bullet}\left(X_{n, k}^{(r)}\right)$ vanishes in odd degree. The second is Theorem 3.3. The third follows from the definitions of $S_{n, k}^{(r)}$ and $R_{n, k}^{(r)}$. The fourth follows from Corollary 2.6. The space $X_{n, k}^{(r)}$ of line configurations therefore gives a geometric model for ordered $r$-Stirling partitions. It may be possible to exploit this geometric model to describe the graded structure of $R_{n, k}^{(r)}$ as follows; the authors thank an anonymous referee for pointing this out. 
Let $G(r, k)$ be the Grassmannian of $r$-dimensional subspaces $V \subseteq \mathbb{C}^{k}$ and consider the subspace $Y_{n, k}^{(r)} \subseteq G(r, k) \times\left(\mathbb{P}^{k-1}\right)^{n-r}$ defined as follows

$$
Y_{n, k}^{(r)}:=\left\{\left(V, \ell_{r+1}, \ldots, \ell_{n}\right): V+\ell_{r+1}+\cdots+\ell_{n}=\mathbb{C}^{k}\right\}
$$

The space $Y_{n, k}^{(r)}$ is an open subvariety of $G(r, k) \times\left(\mathbb{P}^{k-1}\right)^{n-r}$. We have a natural map

$$
\begin{gathered}
\pi: \quad X_{n, k}^{(r)} \longrightarrow Y_{n, k}^{(r)} \\
\left(\ell_{1}, \ldots, \ell_{r}, \ell_{r+1}, \ldots, \ell_{n}\right) \longmapsto\left(\ell_{1}+\cdots+\ell_{r}, \ell_{r+1}, \ldots, \ell_{n}\right)
\end{gathered}
$$

obtained by taking the (necessarily $r$-dimensional) span of the first $r$ lines in a typical configuration in $X_{n, k}^{(r)}$.

The map $\pi: X_{n, k}^{(r)} \rightarrow Y_{n, k}^{(r)}$ is a fiber bundle. The fiber $F$ over a point $\left(V, \ell_{r+1}, \ldots, \ell_{n}\right) \in Y_{n, k}^{(r)}$ is given by the space of $r$-tuples $\left(\ell_{1}, \ldots, \ell_{r}\right)$ of linearly independent lines in the $r$-dimensional vector space $V$, which is homotopy equivalent to the flag variety $\mathcal{F} \ell(r)$. The inclusion $\iota: F \hookrightarrow X_{n, k}^{(r)}$ induces a map on rational cohomology $\iota^{*}: H^{\bullet}\left(X_{n, k}^{(r)} ; \mathbb{Q}\right) \rightarrow H^{\bullet}(F ; \mathbb{Q})$. Since $H^{\bullet}(F ; \mathbb{Q})$ is generated by the Chern classes $c_{1}\left(\ell_{1}^{*}\right), \ldots, c_{1}\left(\ell_{r}^{*}\right)$ of the tautological line bundles $\ell_{1}^{*}, \ldots, \ell_{r}^{*}$ over $F$, and these line bundles are pullbacks under $\iota$ of the corresponding bundles on $X_{n, k}^{(r)}$, the map $\iota^{*}$ is a surjection.

By the last paragraph, the Leray-Hirsch Theorem (see e.g. [4]) provides the following isomorphism of $H^{\bullet}\left(Y_{n, k}^{(r)} ; \mathbb{Q}\right)$-modules:

$$
H^{\bullet}\left(X_{n, k}^{(r)} ; \mathbb{Q}\right) \cong H^{\bullet}(F ; \mathbb{Q}) \otimes_{\mathbb{Q}} H^{\bullet}\left(Y_{n, k}^{(r)} ; \mathbb{Q}\right) .
$$

The isomorphism (54) seems quite close to the conjectural isomorphism (33). The left-hand-side of (54) is the graded $S_{r} \times S_{n-r}$-module $R_{n, k}^{(r)}$. The tensor factor $H^{\bullet}(F ; \mathbb{Q})$ is the classical coinvariant module $R_{r}$ for the symmetric group $S_{r}$. Determining the graded $S_{r} \times S_{n-r}$-isomorphism type of $R_{n, k}^{(r)}$ therefore reduces to determining the graded $S_{n-r^{-}}$structure of $H^{\bullet}\left(Y_{n, k}^{(r)} ; \mathbb{Q}\right)$.

\section{Acknowledgements}

B. Rhoades was partially supported by NSF Grant DMS-1500838. A. T. Wilson was partially supported by an NSF Mathematical Sciences Postdoctoral Research Fellowship. The authors thank an anonymous referee for 
their careful reading of the paper and, in particular, for pointing out the applicability of the Leray-Hirsch Theorem. We thank Jeff Remmel for his mathematics, mentoring, and friendship.

\section{References}

[1] C. Chevalley. Invariants of finite groups generated by reflections. Amer. J. Math., 77 (4) (1955), 778-782.

[2] J. Haglund, J. Remmel, and A. T. Wilson. The Delta Conjecture. Trans. Amer. Math. Soc., 370 (2018), 4029-4057.

[3] J. Haglund, B. Rhondes, and M. Shimozono. Ordered set partitions, generalized coinvariant algebras, and the Delta Conjecture. Adv. Math., 329 (2018), 851-915.

[4] A. Hatcher. Algebraic Topology. Cambridge: Cambridge University Press. (2002)

[5] B. Pawlowski and B. Rhohdes. A flag variety for the Delta Conjecture. Submitted, 2018. arXiv:1711.08301.

BRENDON RHOADES

Department of Mathematics

University of California, San Diego

LA Jolla, CA, 92093, USA

E-mail address: bprhoades@math.ucsd.edu

ANDREW Timothy Wilson

Department of Mathematics

Portland State University

Portland, OR, 97201, USA

E-mail address: andwils2@pdx.edu

Received January 1, 0000 